\title{
Percepção dos profissionais da saúde acerca da comunicação de más notícias e óbitos no contexto perinatal
}

\author{
Perinatal care professionals' perception of bad news and fetal deaths \\ Percepción de los profesionales de la atención perinatal sobre las malas noticias y las muertes \\ fetales
}

Edilene Eva Garcia da Silva

ORCID: https://orcid.org/0000-0003-4069-9349

Universidade Franciscana, Brasil

E-mail: dillygarcia82@gmail.com

Giovana Cóssio Rodriguez

ORCID: https://orcid.org/0000-0002-5966-6582

Universidade Franciscana, Brasil

E-mail: giovanacossio@gmail.com

Gabriel Bloedow da Silveira

ORCID: https://orcid.org/0000-0002-9633-2063 Universidade Franciscana, Brasil

E-mail: gabrielbloedowds@gmail.com

Thalyta Freitas dos Santos Laguna

ORCID: https://orcid.org/0000-0002-4227-0020 Universidade Fransicana, Brasil

E-mail: thalytalaguna@gmail.com

Maria Luisa Suárez Gutiérrez Cella

ORCID: https://orcid.org/0000-0002-8836-8699 Universidade Franciscana, Brasil

E-mail: malu_suarez@hotmail.com

Rosiane Filipin Rangel

ORCID: https://orcid.org/0000-0003-4059-4176 Universidade Franciscana, Brasil

E-mail: rosianerangel@yahoo.com.br

Cristina Saling Kruel

ORCID: https://orcid.org/0000-0003-1996-7708

Universidade Franciscana, Brasil

E-mail: cristinaskruel@gmail.com

\begin{abstract}
Resumo
Esta pesquisa teve como objetivo investigar as percepções dos profissionais da assistência perinatal quanto à comunicação de más notícias e óbitos no contexto perinatal. Trata-se de uma pesquisa descritiva exploratória, de abordagem quali-quantitativa. Participaram do estudo 27 profissionais da assistência perinatal hospitalar, dentre esses, enfermeiras obstétricas, técnicas de enfermagem, enfermeiras residentes em enfermagem obstétrica, médicos ginecologistas obstetras. A coleta foi feita por meio de questionário com questões organizadas numa escala likert e questões abertas que foram submetidas à Análise de Conteúdo. Os dados revelam a dificuldade de o profissional vivenciar uma perda perinatal e lidar com a comunicação de más notícias. Conclui-se que, de acordo com a percepção dos profissionais da assistência perinatal, os mesmos sentem-se pouco preparados para a comunicação de más notícias e óbitos perinatais.
\end{abstract}

Palavras-chave: Maternidades; Óbito fetal; Gravidez.

\begin{abstract}
This research aimed to investigate the perceptions of perinatal care professionals regarding the communication of bad news and deaths in the perinatal context. This is an exploratory descriptive research, with a qualitative and quantitative approach. Twenty-seven perinatal hospital care professionals participated in the study, among these obstetric nurses, nursing technicians, nurses residing in obstetric nursing, obstetric gynecologists. The collection was made through a questionnaire with questions organized through a likert scale and open questions that were submitted to Content Analysis. The data reveal how difficult it is for professionals to experience perinatal loss and deal with the communication of bad news. It is concluded that in the perception of professionals of perinatal care they feel little prepared for the communication of bad news and perinatal deaths.
\end{abstract}

Keywords: Maternity; Fetal death; Pregnancy. 


\section{Resumen}

Esta investigación tuvo como objetivo investigar las percepciones de los profesionales de la atención perinatal sobre la comunicación de malas noticias y muertes en el contexto perinatal. Se trata de una investigación descriptiva exploratoria, con enfoque cualitativo y cuantitativo. En el estudio participaron 27 profesionales de la atención hospitalaria perinatal, entre estos enfermeras obstétricas, técnicos de enfermería, enfermeras residentes en enfermería obstétrica, ginecólogos obstétricos. La recolección se realizó a través de un cuestionario con preguntas organizadas a través de una escala Likert y preguntas abiertas que fueron enviadas a Análisis de Contenido. Los datos revelan lo difícil que es para los profesionales experimentar una pérdida perinatal y lidiar con la comunicación de malas noticias. Se concluye que en la percepción de los profesionales de la atención perinatal se sienten poco preparados para la comunicación de malas noticias y muertes perinatales.

Palabras clave: Maternidades; Muerte fetal; Embarazo.

\section{Introdução}

Comunicar uma má notícia para o paciente e seus familiares é um grande desafio para os profissionais de saúde, haja vista que a informação terá impacto negativo na experiência das pessoas envolvidas. Trata-se, então, de uma condição delicada, que exige atenção às particularidades daquilo que precisa ser comunicado, da pessoa e dos familiares que receberão a notícia. A maneira como a má notícia é transmitida repercute nas reações imediatas das pessoas envolvidas e no desenrolar da elaboração mental frente ao evento adverso (Pereira, 2005).

Diferentemente de áreas de atenção em saúde que, de maneira geral, relacionam-se às emergências e ao adoecimento e seus desdobramentos, os profissionais da assistência perinatal atuam com eventos predominantemente saudáveis, esperados e desejados. Portanto, as intercorrências e a morte são inesperadas e exigem dos profissionais um manejo especialmente complexo (Setubal, 2016). Entre as más notícias que são comunicadas pelos profissionais da assistência perinatal, o óbito perinatal está entre mais difíceis, especialmente porque o parto e o nascimento são eventos atrelados à saúde e ao começo da vida. Há, nesses casos, uma inversão do ciclo da vida que é interrompida logo em seu começo (Setubal, 2018).

Nomeia-se óbito fetal aquele que ocorre no período entre a $22^{\mathrm{a}}$ semana completa de gestação, ou 154 dias anterior ao nascimento do bebê, ou de fetos com peso igual ou superior a 500g ou estatura a partir de $25 \mathrm{~cm}$ (RIPSA, 2008). Já os óbitos perinatais são aqueles que ocorrem entre a $28^{\mathrm{a}}$ semana de gestação e o $7^{\mathrm{o}}$ dia após o nascimento (Bireme - DeCS, 2010). Ademais, óbitos neonatais são aqueles em que o bebê possui até 6 dias de vida, considerado óbito neonatal precoce ou quando a sua idade está entre 7 e 27 dias de vida, chamado de óbito neonatal tardio (Gaiva, Bittencourt e Fujimori, 2013). Os óbitos no contexto perinatal são uma realidade expressiva: aproximadamente 2,8 milhões de bebês morrem em todo o mundo todos os anos durante o primeiro mês após o nascimento e a maioria desses casos acontece em países em desenvolvimento, como o Brasil (Liu et al, 2015).

Os óbitos perinatais também incluem as mortes maternas, que se definem pela morte de uma mulher durante a gestação ou até 42 dias após o término da gestação, causada necessariamente por qualquer fator relacionado à gravidez ou por medidas relacionadas a mesma (Brasil, 2009). A mortalidade materna ainda é alta em várias regiões do mundo. Segundo a Organização Pan-Americana de Saúde (OPAS), cerca de 830 mulheres morrem todos os dias por complicações relacionadas à gravidez ou ao parto. Estima-se que, em 2015, cerca de 303 mil mulheres morreram durante e após a gravidez e o parto (OPAS, 2018).

Assistir a um óbito fetal ou perinatal é uma experiência desafiadora para profissionais de saúde. Saber como comunicar, respeitar e acolher os familiares é um conhecimento essencial para a promoção de um cuidado humanizado em saúde. Para isto, é necessário não somente ter conhecimento acerca das demandas, configurações e particularidades do luto perinatal, mas também possuir um planejamento e preparo técnico que possa guiar os profissionais através de um evento tão desafiante como é o manejo de um óbito nesse contexto.

Nos últimos anos, observa-se um maior investimento em processos formativos para profissionais da saúde que atuam 
em instituições hospitalares. Porém, os desafios hoje enfrentados pelos mesmos sugerem que os cursos de formação devem repensar seu modo de concepção diante desse assunto, contribuindo para uma preparação mais qualificada. Os progressos nesse âmbito são necessários e devem acontecer nas instituições hospitalares e de ensino, provocando reflexões que possam favorecer uma assistência adequada diante da adversidade (Costa \& Lima 2005).

Destaca-se a importância da existência de uma formação positiva para a comunicação de más notícias de uma maneira geral. Porém, observam-se lacunas quanto à formação e orientação nesse sentido para a área da perinatalidade, evidenciando uma vulnerabilidade muito grande diante de um acontecimento que requer o maior nível de preparo, delicadeza e qualificação (Monteiro et al, 2011).

Sendo assim, torna-se imprescindível discutir sobre a humanização nos processos de comunicação de más notícias em contexto perinatal. O contexto do luto perinatal, em especial, demanda uma atenção qualificada e empática, tratando os sujeitos envolvidos a partir de uma escuta atenta às singularidades e demandas que se apresentam, garantindo um cuidado constante $\mathrm{e}$ também multiprofissional (Brasil, 2013).

Frente a uma situação difícil na perinatalidade, os profissionais necessitam de um preparo sólido para que o manejo deste desafio se dê da forma mais benéfica possível para as famílias. A conduta profissional adequada nesse cenário pode contribuir significativamente para uma elaboração e enfrentamento saudável por parte de pacientes e famílias. Para que haja esse preparo, antes é necessário compreender o processo formativo dos profissionais da saúde quanto a comunicação de más notícias e óbitos perinatais e as suas percepções quanto às habilidades e competências que já possuem.

Sendo assim, objetiva-se com o presente estudo investigar as percepções dos profissionais da assistência perinatal quanto à comunicação de más notícias e óbitos no contexto perinatal.

\section{Metodologia}

Trata-se de um estudo descritivo exploratório de abordagem quanti-qualitativa. Nesse sentido, entende-se que a aliança entre os aspectos quantitativo e qualitativo favorece uma conversa ampla entre o embasamento teórico, os aspectos levantados em números e os âmbitos da realidade que não podem ser quantificados (Gil, 2019; Pereira, 2018; Severino, 2016). Assim, foram convidados a participar da pesquisa um total de 40 profissionais da assistência perinatal de um hospital de médio porte do interior do estado do Rio Grande do Sul. A coleta de dados foi realizada no mês de dezembro de 2020, por meio de um questionário contendo nove questões semiestruturadas e 18 questões estruturadas, que versavam sobre a percepção dos profissionais quanto a sua experiência profissional no contexto de comunicação de más notícias e de óbitos perinatais, além de informações quanto à formação e tempo de serviço. As respostas das questões estruturadas foram organizadas por meio de uma escala likert com as opções "Sim, efetivamente", "Sim, mas pode melhorar" e "Não. Encerrou-se a coleta no momento em que os resultados possibilitaram à pesquisadora compreender as percepções dos profissionais de saúde à frente da saúde maternoinfantil. Participaram da pesquisa um total de 27 profissionais de quatro diferentes ocupações profissionais. Foram usados como critérios de inclusão: Profissionais de saúde formados que atuem diretamente na maternidade de risco habitual. Profissionais que façam parte do quadro fixo de funcionários e/ou contratados do setor da maternidade. E como critérios de exclusão: Profissionais afastados do serviço por férias e/ou licença. Profissionais que atuem no setor a menos de 3 meses (por não estarem efetivados nesse período). As Tabelas 1 e 2 demonstram a caracterização dos participantes da pesquisa, quanto à ocupação profissional e ao tempo de experiência. 
Tabela 1. Caracterização dos participantes da pesquisa quanto à ocupação profissional.

\begin{tabular}{lcc}
\hline \multicolumn{1}{c}{ Cargo } & Total \% & Frequência \\
\hline Enfermeira Obstetra (EO) & $11,11 \%$ & 03 \\
Enfermeira Residente em & $37,04 \%$ & 10 \\
Enfermagem Obstétrica (EREO) & & 05 \\
Médico Obstetra & $18,52 \%$ & 09 \\
Técnico em Enfermagem & $33,33 \%$ & 27 \\
Total & $100,00 \%$ & \\
\hline
\end{tabular}

Fonte: Autores, conforme dados da pesquisa (2020).

Tabela 2. Caracterização dos participantes quanto ao tempo de experiência.

\begin{tabular}{lll}
\hline Tempo de Experiência & $\%$ & Frequência \\
\hline Até 1 ano & $25,93 \%$ & 07 \\
De 1 a 5 anos & $48,15 \%$ & 13 \\
De 5 a 10 anos & $14,81 \%$ & 04 \\
De 10 anos ou mais & $11,11 \%$ & 03 \\
Total & $100 \%$ & 27 \\
\hline
\end{tabular}

Fonte: Autores, conforme dados da pesquisa (2020).

Os achados da pesquisa foram submetidos à Análise de Conteúdo (Bardin, 2016) em que se verificou a frequência das respostas quantificáveis e as particularidades e semelhanças entre as respostas às perguntas abertas. Quanto às perguntas cujas respostas foram coletadas por meio da escala likert, os dados foram expostos na tabela abaixo.

As questões éticas foram respeitadas de acordo com a Resolução 466/2012. O projeto recebeu aprovação pelo Comitê de Ética em Pesquisa da Universidade Franciscana (UFN) sob o número CAAE 40196520.0.0000.5306. Para garantia de sigilo aos participantes, foram atribuídos códigos conforme a profissão e ordem de resposta, sendo Enfermeiro Obstetra - EO, Enfermeiro Residente em Enfermagem Obstétrica - EREO, Médico Obstetra - MO e Técnico em Enfermagem - TE.

\section{Resultados e Discussão}

Observou-se que a maioria dos profissionais tem a percepção de que pode melhorar o preparo na abordagem com famílias em processo de luto perinatal. Também se observa que os profissionais entrevistados não se sentem capacitados para cuidar das famílias frente a uma perda perinatal. Notou-se que os envolvidos relatam pouco preparo em comunicar um óbito perinatal. A maioria dos profissionais respondeu que entende a diferença entre óbito fetal, perinatal e neonatal. Percebe-se que os profissionais se sentem pouco preparados em atender uma gestante portadora de alguma complicação gestacional. Observase que a maioria dos profissionais acredita que a abordagem com a mulher pode melhorar, diante de uma transferência de RN ao UTI/Neonatal após o nascimento, conforme demostrado na Tabela 3. 
Tabela 3. Resultados referentes à percepção dos profissionais quanto ao seu conhecimento, preparo e capacidade para atuar em contexto de comunicação de más-notícias e lutos perinatais.

Me sinto preparada(o) para abordar/dialogar com famílias em processo de luto perinatal.

\begin{tabular}{lcc}
\hline Preparação dos profissionais & N & $\%$ \\
\hline Sim, mas pode melhorar & 16 & 59,26 \\
Não & 11 & 40,74
\end{tabular}

Me sinto capacitada(o) para proceder e cuidar dos envolvidos durante uma perda perinatal.

\begin{tabular}{lcc}
\hline $\begin{array}{l}\text { Capacitação diante } \\
\text { da perda }\end{array}$ & N & $\%$ \\
\hline Sim, efetivamente & 1 & 3,7 \\
Sim, mas pode melhorar & 15 & 55,56 \\
Não & 11 & 40,74
\end{tabular}

Me sinto capacitada(o) para proceder e cuidar dos envolvidos durante uma perda materna.

\begin{tabular}{lcc}
\hline Proceder e cuidar & N & $\%$ \\
\hline Sim, mas pode melhorar & 10 & 37,4 \\
Não & 17 & 62,96
\end{tabular}

Me sinto capacitada(o) para comunicar a morte perinatal a uma família é igual a comunicar qualquer outra morte.

\begin{tabular}{lcc}
\hline $\begin{array}{l}\text { Comunicar uma } \\
\text { morte perinatal }\end{array}$ & N & $\%$ \\
\hline Sim, mas pode melhorar & 2 & 7,41 \\
Não & 25 & 92,59
\end{tabular}

Entendo a diferença entre o óbito fetal, perinatal e neonatal.

\begin{tabular}{lcc}
\hline Diferença & N & $\%$ \\
\hline Sim, efetivamente & 17 & 62,96 \\
Sim, mas pode melhorar & 8 & 29,63 \\
Não & 2 & 7,41
\end{tabular}


Comunico a morte perinatal para a mãe com a mesma atenção que comunico para o pai.

\begin{tabular}{lcc}
\hline Comunicar pai e mãe & N & $\%$ \\
\hline Sim, efetivamente & 9 & 33,33 \\
Sim, mas pode melhorar & 9 & 33,33 \\
Não & 9 & 33,33
\end{tabular}

Me sinto capacitada(o) para atender uma gestante portadora de alguma complicação que possa interferir a gestação.

\begin{tabular}{lcc}
\hline Complicação na gestação & N & $\%$ \\
\hline Sim, efetivamente & 5 & 18,52 \\
Sim, mas pode melhorar & 16 & 59,26 \\
Não & 6 & 22,22
\end{tabular}

Sei como proceder com relação a mulheres que têm seus bebês transferidos a uma UTI/Neonatal após o nascimento.

\begin{tabular}{lcc}
\hline Bebês transferidos & N & $\%$ \\
\hline Sim, efetivamente & 1 & 3,7 \\
Sim, mas pode melhorar & 20 & 74,07 \\
Não & 6 & 22,22 \\
\hline
\end{tabular}

Fonte: Autores, conforme dados da pesquisa (2020).

Considerando os dados coletados nas entrevistas, entende-se que as falas dos entrevistados sobre a comunicação de más notícias e óbitos perinatais evidenciam a sua angústia diante das situações ruins. Desse modo, essas falas foram organizadas em duas categorias, que serão discorridas adiante: desafios durante a prática e estratégias para o enfrentamento.

\subsection{Desafios durante a prática}

Profissionais da perinatalidade precisam ser treinados para casos que necessitem lidar com o luto e sua comunicação, a fim de possibilitar boas práticas na assistência à perda perinatal. É necessário diretrizes de intervenção e meios de apoio aos pais e também aos profissionais inseridos nesses contextos (Martínez-Serrano et al, 2018). Para a equipe de enfermagem, a escuta e o suporte emocional são importantes e quanto a isso, um dos participantes do estudo ressaltou a dificuldade que a profissão tem de lidar com situações que demandam de suporte emocional.

'Já presenciei casos de abortamento, onde foram necessários o acolhimento, a escuta e o suporte emocional da mulher e família. Nestas situações o profissional enfermeiro é muito importante, visto que possui um papel de protagonismo do cuidado. Ao meu ver, nós enfermeiros ainda temos muita dificuldade para lidar com situações que demandam suporte emocional, são questões muito particulares, que fogem ao tecnicismo e necessitam de tecnologias e processos de trabalho bem desenvolvidos." (EROE04).

Diante de um óbito gestacional, é fundamental que os profissionais de saúde estejam adequadamente instruídos e capacitados para lidar da melhor maneira possível com a família que se encontra naquela situação, com objetivo de diminuir ou 
reconhecer fatores de risco que contribuem para o desenvolvimento de condições psicopatológicas (Moura, 2015). A importância do ambiente, do atendimento acolhedor e multidisciplinar também foi destacado pelos profissionais participantes.

"Informação... Acolhimento... Empatia... Carinho ... Apoio de outros profissionais.. Equipe multidisciplinar... " (MO1)

"Cuidar para que a paciente não tenha tanto contato com as outras pacientes cujos filhos nasceram bem e saudáveis e dar apoio emocional." (TE4)

Os profissionais envolvidos na assistência perinatal, mencionam sentimentos de tristeza e impotência diante da prática, disponibilizar um espaço para a elaboração da situação vivenciada na assistência em uma perda perinatal necessita ser uma pauta institucional, a qual englobe toda a equipe. $\mathrm{O}$ atendimento multidisciplinar pode favorecer a assistência ao paciente, bem como auxiliar os profissionais em os conflitos que surgem ao vivenciarem a perda gestacional (Lemos \& Cunha, 2015). Os relatos evidenciaram que os profissionais sentem a necessidade de que esses assuntos sejam abordados durante a formação. Ressaltam ainda a dificuldade em vivenciar uma perda perinatal.

'É um momento extremamente delicado tanto para a família que recebe a noticia, quanto para o profissional que irá dar a notícia. Pois, infelizmente, durante a graduação não somos orientados, assim como não existem disciplinas que abordem o tema de uma forma mais profunda. Minha experiência foi chocante, por se tratar de uma gestação a termo, ver que a paciente teve muita dificuldade de assimilar o que estava acontecendo, e quando fui para casa, ficou um vazio, um sentimento de frustração, incapacidade." (EROE03).

Diante do momento de um mau prognóstico na maternidade, percebeu-se na fala dos participantes o quanto os profissionais sentem-se fragilizados e sofrem diante dessas situações.

"Foi horrivel, pois ninguém quer vivenciar um momento que é de felicidade para todas familia com tudo pronto para esperar em casa e você falar que isso não vai acontecer. Sofri junto com a família é muito triste." (EO3)

Estudos mostram que os profissionais sofrem com a morte, de maneira semelhante à família, interferindo emocionalmente em suas vidas, sendo assim, buscam meios de enfrentamento do luto, a fim de conviver com a perda (Silva et al, 2015).

Profissionais necessitam de apoio psicológico para vivenciar e enfrentar o luto dos seus pacientes. A inclusão do tema nos currículos mostra-se um aliado no enfrentamento, bem como estratégias de educação permanente com esse assunto, promovendo mudanças de comportamento em relação ao luto vivenciado e as frustrações causadas diante de uma perda (Costa \& Lima, 2005). Quando questionados sobre o tipo de manejo realizado diante de uma complicação durante a gestação, os profissionais relatam suas vivências.

"Orientação sobre a doença e as possíveis repercussões para o feto de forma clara e me colocando a disposição para conversar sobre sempre que achar necessário. Além disso apoiar a mulher nesse processo difícil." (EREO1)

" Orientações sobre o tipo de doença e as medidas para reduzir os danos ao bebe. Uma postura acolhedora sem julgamentos." (EREO2)

"Gestante encaminhada a um ambiente privativo, calmo e acolhedor, na presença somente dos profissionais 
responsáveis pela realização de testes ou exames que tiveram resultado alterado, comunicado separadamente do familiar que estiver junto, preservando o direito de escolha em contar ou não e preservando a privacidade da gestante." (EREO7)

A comunicação de más notícias é uma das atividades mais complicadas na prática clínica dos profissionais e não existe preparação consistente na formação acadêmica. Programas de treinamento mostram-se bons aliados na lacuna existente entre a formação e a prática, a fim de melhorar a comunicação entre paciente e suas famílias (Victorino, et al, 2007). O modo que será comunicado a má notícia influência na reação do paciente e nas famílias, por isso a importância de uma abordagem adequada e a prática humanizada nesses casos, bem como, habilidades que favoreçam o entendimento, o qual fará diferença no decorrer do processo (Fontes et al, 2017). Percebe-se nos relatos como é difícil pra equipe trabalhar com a comunicação de más notícias, precisando lidar com suas próprias frustrações e angústias diante de um contexto assim.

'’ Sim, inúmeras más notícias [...] Desde abortamento até indicação de alguma cesariana de urgência [...] Sempre é difícil [...] Sempre tentamos fazer o melhor pela mãe e o bebê [...] muitas vezes lidamos com a frustração da família, da equipe e nossa também,' (MO1)

' 'Prestei cuidados após uma paciente chegar na unidade encaminhada de outro hospital, quando chegou na unidade, após 2 horas de ser aceita pelo médico plantonista do dia, não foi conseguido auscultar o BCF do feto e a gestante foi encaminhada para uma cesariana de emergência, eu estive presente na cesariana após o nascimento foi tentando reanimar porém sem sucesso [...] Acompanhei a paciente na sala de recuperação uma jovem moça que se encontrava inconsolada, prestei meus cuidados técnicos e meus emocionais, nessas horas não sabemos o que falar, como confortar uma mãe que planejou esse filho(a), que cuidou de cada detalhe do quartinho das roupinhas, nós profissionais não temos preparação e nem frieza para lidar com esse sentimento de que somos importantes em um monte tão frágil e triste para uma mulher que teve seus sonhos devastados pelo destino.", (TE4)

Um estudo realizou a comparação entre mulheres do Brasil e do Canadá, o qual verificou a associação do luto complicado nessas mulheres. A pesquisa identificou que as mulheres com maior comprometimento do estado de luto são as Brasileiras, as quais não integram grupo de apoio, sem prática de religião e com menor nível de escolaridade (Paris et al, 2016).

De acordo com Pereira (2009), notícias ruins devem ser divididas e não dadas, a fim de possibilitar ao paciente e seus familiares assimilarem questões expostas, sendo dirigidos para a verdade. Comunicar más notícias é uma das atribuições mais difíceis na atuação profissional, e os profissionais reconhecem isso, evidenciando que é impossível evitá-la durante a prática. Desta forma, a comunicação de más notícias pode ser mais branda através de técnicas e treinamento, referentes a profissão, facilitando a atuação.

Para transmitir a má notícia, o profissional precisa conhecer as famílias, ter o máximo de cuidado com o local, o momento e como dará a notícia. A família precisa estar junto ao serem informados. Essas ações são traçadas no plano de atendimento, assim como as estratégias do decorrer dos casos, revendo a necessidade de cada situação (Ponte et al, 2012). São inúmeros os desafios descritos pelo profissional, diante de uma perda perinatal, surgindo conflitos que geram ansiedade e angústia em toda a equipe.

“' Essa situação é só desafios, acredito que ninguém está pronto para isso. O desafio de ver uma família em formação acabar, os sonhos da família serem tirados dela, é muito difícil [...] Toda expectativa e espera pelo novo membro da 
família, ter que lidar com tudo isso é muito difícil, tanto para família como para os profissionais. E essa busca por culpados, de apontar para alguém e colocar toda a culpa naquela pessoa, se tornar um vilão. Em um momento em que tu só quer fazer o bem. Além de ver muitas vezes a família se culpando (quando a morte ocorre intra útero) ou culpando outros colegas. É um momento muito delicado e dificil" (MO1)

"A reação de toda família frente ao luto e ter que dar suporte para a equipe, pois toda a equipe fica abalada com toda a situação." (EO3)

Percebe-se ser essencial explorar os sentimentos gerados pela subjetividade dos profissionais diante do contexto vivenciado. Em estudo realizado por Silva et al (2010), os pesquisadores evidenciaram que os sentimentos dos profissionais diante da perda foram emergindo de forma lenta, manifestando fragilidade, bem como, em que os profissionais demonstraram sentir fracasso, impotência, com momentos de força e coragem frente ao sentimento da família.

\subsection{Estratégias para o enfrentamento}

Uma pesquisa realizada sobre o conhecimento dos profissionais de saúde antes e depois de um programa educacional sobre luto perinatal identificou que a percepção dos profissionais quanto as necessidades de cuidados emocionais das famílias, aumentou após um programa educacional. Esse mesmo estudo identificou que os profissionais não consideram o aborto uma perda tão expressiva quanto ao natimorto e a perda neonatal (Dimarco et al, 2002).

Os profissionais entrevistados mostraram a necessidade que sentem em capacitações acerca do assunto de luto perinatal e comunicação de más notícias, em que favorece o cuidado ao paciente e suas famílias, humanizando a assistência e colaborando para o enfrentamento com essas situações. Quando questionados sobre estratégias sugeridas para lidar em situações de luto perinatal e comunicação de más notícias, alguns deles sugerem capacitações da equipe e realização de rituais de despedida.

\footnotetext{
“Falarmos mais sobre o assunto através de capacitações, que muitas vezes é deixado de lado." (EREO4)

" Capacitações de más notícias, capacitações profissionalizantes de fatores que podem levar isso a acontecer." (EREO7)

"Preparar rituais de despedida e preparo de material de lembrança da criança como carimbo do pé, mecha de cabelo, distanciar os familiares de outras famílias com RN no momento inicial." (EO1)
}

Evidenciou-se na fala dos entrevistados que estratégias para o enfrentamento da temática precisam ser (re)pensadas, pois por mais que ninguém queira/espere esse desfecho, pode acontecer. Dentre essas, entende-se que o pré-natal seria um momento possível para discussão do assunto.

"Muito difícil pensar em uma estratégia [...]. Acho que um pré-natal com bastante informação sobre tudo que envolve uma gestação seria o melhor. Porém, o ser humano acha que nunca as coisas ruins vão acontecer com ele [...] Então é uma tarefa muito difícil a aceitação e o preparo... "' (MO1)

Diante das falas dos profissionais, percebe-se a dificuldade em lidar com situações que não são esperadas durante o cenário de nascimento, bem como, perda perinatal e más notícias. O comprometimento emocional nesses casos é notável nas falas. Foram identificados no presente estudo sentimentos como frustração e impotência, pelo fato de não conseguir evitar certos acontecimentos durante a assistência perinatal, os quais fogem do esperado em uma gestação. 
A partir dos estudos fica evidente a necessidade de atualizar os currículos universitários e técnicos da formação em saúde, objetivando a adequada prática dos profissionais nos casos de óbito fetal. Exige-se novos modelos assistenciais com identificação prévia dos casos de luto, a fim de desenvolver habilidades profissionais conduzindo para um atendimento apropriado nas necessidades na área do luto (Paris et al, 2017).

\section{Conclusão}

Considera-se que o objetivo do estudo foi alcançado, pois conseguiu-se identificar que profissionais da assistência perinatal se sentem pouco preparados para a comunicação de más notícias e óbitos perinatais. Os resultados numéricos e qualitativos sugerem que esse é um tema que merece ser discutido nas equipes de saúde e deve fazer parte de processos de educação permanente em saúde, visto que é um assunto que exige estratégias institucionais, visando melhor enfrentamento, assim como uma assistência qualificada.

Pretende-se, assim, contribuir para a compreensão de demandas e formação de um conhecimento sólido acerca do tema, o que pode favorecer uma movimentação social, profissional e científica que procure transformar a realidade da atenção em saúde perinatal.

Evidencia-se a importância de capacitações e momentos de reflexões que possibilitem aos profissionais oportunidades de fala e escuta, proporcionando condições para que os mesmos sejam ouvidos. Ações como essas favorecem a assistência perinatal, oportunizando que os envolvidos nesse processo tenham a oportunidade de expor suas angústias, assegurando o enfrentamento diante de um óbito perinatal ou outra adversidade que pode estar presente na prática. Acredita-se que, por meio dessa pesquisa, os profissionais consigam realizar a reflexão das principais dificuldades diante de uma perda perinatal e a comunicação de más notícias durante a prática assistencial, proporcionando a criação de rotinas assistenciais a fim de melhorar o enfrentamento.

Sugerem-se novos estudos na área que busquem meios de enfrentamento ao luto de profissionais da assistência perinatal e a comunicação de más notícias. Além disso, pode-se pensar em estratégias que favoreçam os processos formativos de profissionais da saúde quanto ao manejo da comunicação de más notícias e óbitos perinatais. A construção e distribuição de guias, cartilhas e outros materiais instrucionais, pode proporcionar o maior alcance dessas informações junto às equipes de assistência perinatal em território nacional.

\section{Referências}

Bardin, L. (2016). Análise de Conteúdo. Edições 70.

Brasil. (2013). Ministério da Saúde. Política Nacional de Humanização - HumanizaSUS.

Brasil, Ministério da Saúde, Secretaria de Atenção à Saúde, Departamento de Ações Programáticas Estratégicas. (2009). Manual dos comitês de mortalidade materna. (3a ed.), Editora do Ministério da Saúde.

Bireme. Descritores em Ciências da Saúde - DeCS. http://decs.bvs.br/P/decs2010p.htm

Costa, J. C. D. \& Lima, R. A. G. (2005). Luto da equipe: revelações dos profissionais de enfermagem sobre o cuidado à criança/adolescente no processo de morte e morrer. Rev. Latino-Am. Enfermagem, 13(2).

DiMarco, M., Renker, P., Medas, J., Bertosa, H. \& Goranitis, J. L. (2002). Effects of an educational bereavement program on health care professionals' perceptions of perinatal loss. J Contin Educ Nurs, 33(4):180-6.

Fontes, C. M. B., Menezes, D. V., Borgato, M. H., \& Luiz, M. R. (2017). Communicating bad news: an integrative review of the nursing literature. Rev Bras Enferm, 70(5):1089-1095. 10.1590/0034-7167-2016-0143.

Gaiva, M. A. M., Bittencourt, R. M. \& Fujimori, E. (2013). Óbito neonatal precoce e tardio: perfil das mães e dos recém-nascidos. Revista Gaúcha de Enfermagem, 34(4):91-97.

Gil, A. C. (2019). Como elaborar projetos de pesquisa (6a ed.). Atlas. 
Lemos, L. F. S. \& Cunha, A. C. B. (2015). Morte na maternidade: como profissionais de saúde lidam com a perda. Psicologia em Estudo, 20(1). Departamento de Psicologia - Universidade Estadual de Maringá. <https://www.redalyc.org/jatsRepo/2871/287142227003/html/index.html>

Liu, L., Oza, S., Hogan, D., Perin, J., Rudan, I., Lawn, J. E. et al. (2015). Global, regional, and national causes of child mortality in 2000-13, with projections to inform post-2015 priorities: an updated systematic analysis. The Lancet. 385(9966): 430-440. <https://www.thelancet.com/journals/lancet/article/PIIS01406736(14)61698-6/fulltext>

Monteiro, S. M., Sánchez, J. M. R., Montoro, C. H., Crespo, M. L., Jaén, A. G. V. \& Tirado, M. B. R. (2011). A experiência da perda perinatal a partir da perspectiva dos profissionais de saúde. Rev. Latino-Am. Enfermagem 19(6).

Martínez-Serrano, P., Palmar-Santos, A. M., Solís-Muñoz, M., Álvarez-Plaza, C. \& Pedraz-Marcos, A. (2018). Midwives' experience of delivery care in late foetal death: A qualitative study. Midwifery. 66:127-133. 10.1016/j.midw.2018.08.010.

Moura, E.C.M. (2015). Vivências de mulheres em situação de abortamento. 63 f. Dissertação (Mestrado em Enfermagem) - Escola de Enfermagem e Farmácia, Programa de Pós-Graduação em Enfermagem, Universidade Federal de Alagoas, Maceió.

OPAS, Organização Pan-Americana de Saúde. (2018). Folha Informativa - Mortalidade Materna. OPAS Brasil. $<$ https://www.paho.org/bra/index.php?option=com_content\&view=article\&id=5741:folha-informativa-mortalidade-materna\&Itemid=820 >

Paris, G. F., Montigny, F. \& Pelloso, S.M. (2016). Factors associated with the grief after stillbirth: a comparative study between Brazilian and Canadian women. Rev Esc Enferm USP. 50(4):546-553. 10.1590/S0080-623420160000500002. PMID: 27680038.

Paris, G. F., Montigny, F., Pelloso, S. M. (2017). Adaptação transcultural e evidências de validação da perinatal grief scale. Cogitare Enfermagem, 26(1).

Pereira, A. S., Shitsuka, D. M., Parreira, F. J., \& Shitsuka, R. (2018). Metodologia da pesquisa cientifica. UFSM. https://repositorio.ufsm.br/bitstre am/handle/1/15824/Lic_Computacao_Metodologia- Pesquisa-Cientifica.pdf?sequence=1

Pereira, M. (2005). Má notícia em saúde: um olhar sobre as representações dos profissionais de saúde e cidadãos. Texto contexto - enferm, 14(1), p. 33-37. 2005 .

Pereira, V. (2009). Comunicar o fim de vida... o papel do enfermeiro face à comunicação de falecimento à família. Revista Enformação [Internet]. 12:14-7. http://repositorio.chlc.minsaude.pt/bitstream/10400.17/176/1/Enforma\%C 3\%A7\%C3\%A3o\%202009\%2014.pdf

Ponte, J., Perpiñán, S., Mayo, M. E., Millá, M. G., Pegenaute, F. \& Poch-Olivé, M. L. (2012). Estudio sobre los procedimientos profesionales, las vivencias y las necesidades de los padres cuando se les informa de que su hijo tiene una discapacidad o un trastorno del desarrollo. La primera noticia. Rev Neurol. 54(Suppl 1):S3-9.

RIPSA, Rede Interagencial de Informação para a Saúde. (2008). Indicadores básicos para a saúde no Brasil: conceitos e aplicações. (2a ed.), Organização Pan-Americana da Saúde.

Setubal, M. S. V. (2016). Avaliação de um programa de treinamento para residentes na comunicação de más notícias em perinatologia utilizando pacientes simulados: ensaio controlado e aleatorizado. [tese de doutorado]. Universidade Estadual de Campinas: http://repositorio.unicamp.br/bitstream/REPOSIP/321591/1/Setubal_MariaSilviaVellutini_D.pdf

Setubal, M. S. V. et al. (2018). Improving perinatology residents' skills in breaking bad news: A randomized intervention study. Revista Brasileira de Ginecologia e Obstetricia, 40(3), 137-146.

Severino, A. J. (2016). Metodologia do trabalho científico (24a ed.). Cortez.

Silva, A. F., Issi, H. B., Motta, M. G. C. \& Botene D. Z. (2015). Cuidados paliativos em oncologia pediátrica: percepções, saberes e práticas na perspectiva da equipe multiprofissional. Rev Gaucha Enferm, 36(2):56-62. 10.1590/1983-1447.2015.02.46299.

Silva, L. C. S. P., Valenca, C. N. \& Germano, R. M. (2010). Percepções dos profissionais de enfermagem intensiva frente a morte do recém-nascido. Rev. bras. Enferm. 63(2): 238-242. http://dx.doi.org/10.1590/S0034-71672010000200011.

Victorino, A. B. et al. (2007). Como comunicar más notícias: revisão bibliográfica. Rev. SBPH, 10(1): 53-63. 